\title{
17beta-Hydroxysteroid Dehydrogenase Type 5 Inhibitor ASP9521
}

National Cancer Institute

\section{Source}

National Cancer Institute. 17 beta-Hydroxysteroid Dehydrogenase Type 5 Inhibitor

ASP9521. NCI Thesaurus. Code C133224.

A selective, orally bioavailable inhibitor of 17 beta-hydroxysteroid dehydrogenase type 5

(17bHSD5, aldo-keto reductase 1C3; AKR1C3), with potential antineoplastic activity. Upon administration, ASP9521 selectively binds to and inhibits the activity of 17bHSD5. This prevents the conversion of the adrenal androgens dehydroepiandrosterone and androstenedione into 5-androstenediol and testosterone. By blocking testosterone production, ASP9521 may inhibit the growth of testosterone-dependent cancers such as castration-resistant prostate cancer (CRPC). 17bHSD5, expressed both in normal prostate tissue and in prostate cancer (PC), plays a crucial role in persistent production of androgens despite castration; its expression is associated with increased malignancy of PC. 